\title{
Obtaining of high quality talc from talcose rocks: a case study from the Sinec and Kokava deposits (Slovakia)
}

\author{
JÁN DERCO; ZOLTÁN NÉMETH \\ Geological Survey of Slovak Republic, Jesenského 8, 04001 Košice, \\ Slovak Republic, nemeth@gssr-ke.sk
}

(Received December 2002 Accepted December 2002)

\begin{abstract}

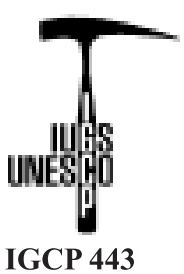

The talcose rocks of lower quality and rarely exploited rock varieties accompanying talc occurrences were tested aiming to obtain talc products of high quality. The main goal was to contribute to wasteless technology of processing, because these rocks are usually stored in dumps and cause environmental threat.

The talc extraction and benefication from carbonatic talcose rocks - talc-magnesite, talcdolomite and talc-magnesite-dolomite was done using flotation method in case study from Sinec deposit. The talc flakes, present in sericite-chlorite schistose rocks in Kokava deposit, were successfully tested for special use in paperboard for roof's covering. Both deposits belong to the strip of talcose occurrences in Veporic unit of Central Slovakia.
\end{abstract}

Key words: talcose rocks, high quality talc, talc flakes, flotation, benefication, wasteless technology, Sinec, Kokava, Veporic unit, Slovakia

\section{Introduction}

The study is targetted to obtain the talc of required industrial quality from talcose rocks accompanying the talc deposits. In the Sinec deposit these were (1) talc-magnesite, (2) talc-dolomite and (3) talcmagnesite-dolomite rock types. The goal was to obtain the high quality talc for pharmaceutical and electrotechnical industry using flotation method. The Kokava deposit with high content of accompanying flaky talcose schists served for obtaining suitable talc flakes for building industry. Studied there were (1) sericite-chlorite schists, (2) steatitized sericitechlorite schists and (3) strongly steatitized chlorite schists.

\section{Geological and tectonic settings of the Sinec and Kokava deposits}

Talc-magnesite deposits are located in distinct strip of mylonitic muscovite-chlorite schists interlayered with metacarbonates and metabasic rocks (Sinec Complex of Lower Carboniferous? age;
Bezák ed. 1999). This strip is sandwitched between Precambrian?-Lower Paleozoic crystalline rocks of Veporic tectonic unit of Western Carpathians (Fig. 1). The garnet-biotite-plagioclase paragneisses, mica schists and large bodies of biotite granodiorites to tonalites prevail northward of the strip. To the south of the strip the older outcropped tectonic étage is built up by biotite-albite gneisses and leucocratic, locally aplitic granites. These in distinct strip trending NESW and crossing through the town Hnúšt'a penetrate the beds of biotite hornfels phyllites. This Pre-Alpine crystalline basement is covered by Carboniferous phyllites and metasandstones as well as Permian arcosic sandstones and conglomerates.

The Pre-Alpine basement and its cover is burried beneath the flat lying body of low-grade muscovitequartz schists, locally with carbonatic matter and interlayers of metacarbonates and metabasics (Sinec Complex, 1.c.). Due to observed regional tectonic discordance and tectonization at the base of the complex there is a vivid discussion that it represents the Alpine overthrusted nappe of Carboniferous Ochtiná Formation of neighbouring south-eastward located Alpine Gemeric 


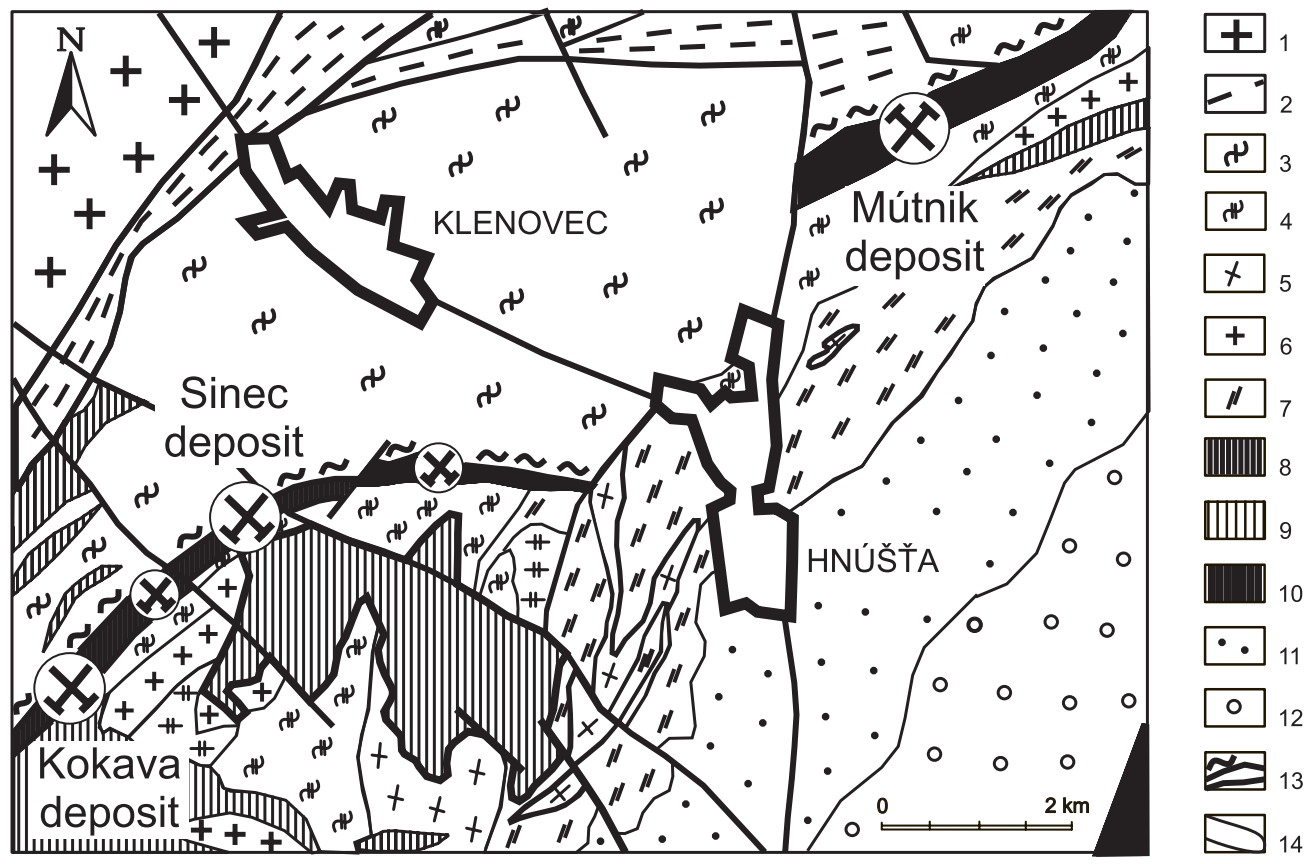

Fig. 1. Position of Sinec and Kokava talc-magnesite deposit in schematic geologic map of south-eastern part of Veporic tectonic unit (simplified after Bezák et al., 1999). 1 - biotite granodiorites to tonalites, locally with bodies of migmatites, orthogneisses of hybridic complex and leucocratic aplitic granitoids and aplites, 2 - mica schists, 3 - garnet-biotite-plagioclase paragneisses, locally amphibolic, 4 - biotite-albite gneisses, locally with bodies of light-coloured fine-grained quartz-feldspar orthogneisses, 5 - migmatites, orthogneisses, granitoids and layers of paragneisses (hybridic complex), local small bodies of leucocratic granites, 6 - leucocratic, locally porphyric granites, granite-porphyries and aplitic granites, 7 - beds of biotite hornfels phyllites to gneisses penetrated with leucocratic to aplitic granites, 1-7 - Proterozoic?-Lower Paleozoic, 8 - muscovite-chlorite schists interlayered with metacarbonates and metabasic rocks, the host rocks of talc-magnesite mineralization, 9 - muscovite-quartz schists locally with carbonatic matter, several small bodies of diorites. 8-9 - Sinec Complex, Lower Carboniferous? representing the nappe outlier? of Gemeric Ochtiná Fm., the main magnesite bearer in the Western Carpathians, 10 - the Gemeric Octiná Fm. schists, metasandstones - it is spread eastward of described area. 11 - phyllites and metasandstones, 12 - arcosic sandstones and conglomerates, 11-12 - Upper Paleozoic cover of Veporic crystalline core. 13 - tectonic boundaries, 14 - lithological transitions.

tectonic unit. The Ochtiná Fm. includes the main occurrences of magnesite mineralization in the Western Carpathians (cf. Grecula et al. 2000 and Radvanec \& Prochaska 2001).

The recent geologic setting of the area is a product of at least two-stadial evolution. The result of tectogenesis of Upper Paleozoic Hercynian era was south-vergent tectonic piling of several crustal units of various lithology and metamorphic evolution (Bezák 1994, Bezák ed. 1999). The origin of first transpressional steeply dipping shear zones is dated back to Hercynian era. It is still unknown whether the former hightemperature metamorphic overprint belongs to Cadomian or old Hercynian tectogenesis (1.c.).

The Alpine (Cretaceous) evolution is characterized by pervasive compression of the territory from the southlying units. There originated the Alpine zonal setting inside the Veporic and Gemeric tectonic units, as well as the Alpine overthrusting of Gemeric unit on Veporic one. In schematic section it is generalized by overthrusting of rocks of the Gemeric Ochtiná Fm. (in low right corner of Fig. 1) on cover sequences of Veporic crystalline basement. The very probable (being still in the state of detail observation) is continuation of this body to outliers of the Sinec Complex west of Hnúšt'a town. Part of Sinec complex was later sandwitched between Veporic crystalline blocks during younger north-vergent transpressional imbrication of the area. This very distinct generally SW-NE driven transpressional strip of so-called Sinec shear zone contains numerous magnesite lenses with partial or full reworking to talc. The only deposit being recently exploited in this strip is the Mútnik deposit located in its NE segment. In other magnesite-talc occurrences, also with abandoned Kokava and Sinec deposits in its SW segment, the exploitation already ceased. 


\section{Talcose rocks from Sinec deposit}

The rock types tested for obtaining of high quality talc can be divided into (1) talc-magnesite, (2) talcdolomite and (3) talc-magnesite-dolomite types.

The rocks were subject of mineralogical and chemical methods of study (Derco 1986). In single rock types participates following minerals: talc, magnesite, chlorite (sheridanite), dolomite, quartz, felspars, muscovite, pyrite and apatite. The distribution of the main rock-forming minerals in the deposit points to a clear decrease of muscovite, quartz and chlorite contents with growing content of talc (Figs. 2 and 3).

Laboratory tests aimed the gaining of high quality talc for electroceramic or pharmaceutical uses. Single samples were prepared by crushing, grinding, homogenization and quartation. The results of subsequent flotation treatment are expressed in Tables 1-4. The best results were obtained using emulsion-kerosene-pineoil

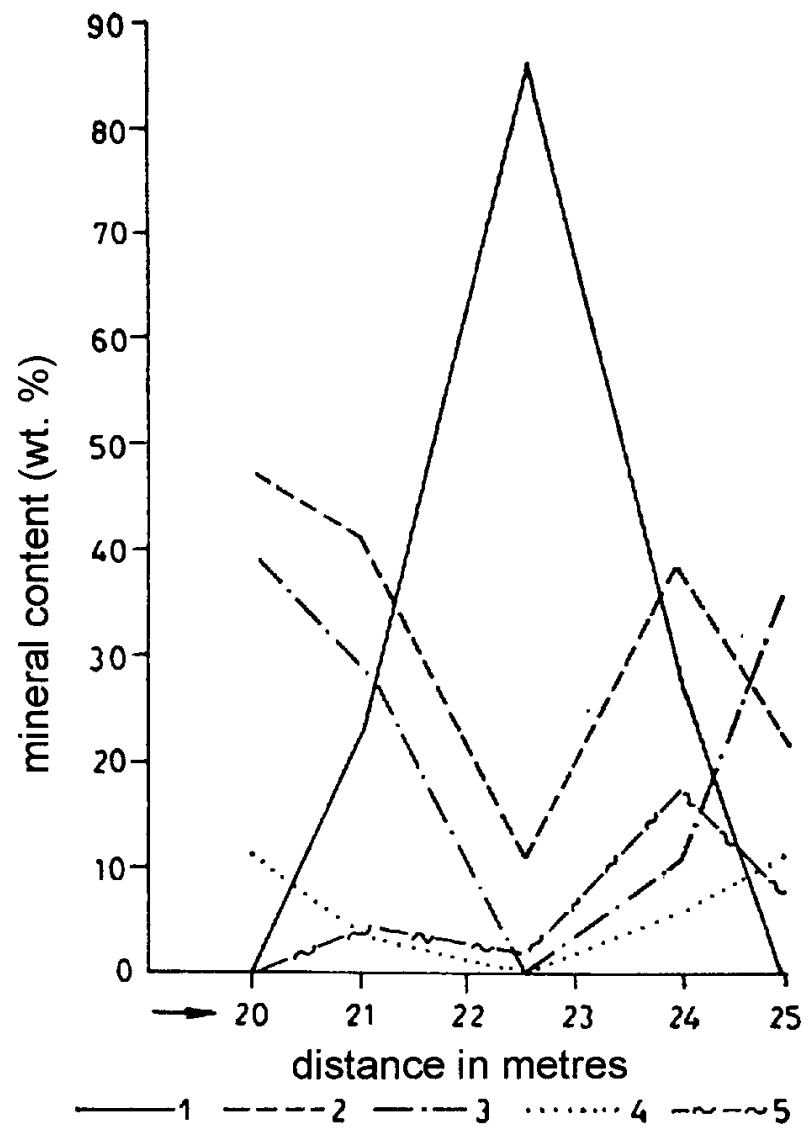

Fig. 2. Distribution of the main mineral components of the deposit in relation to the rock type in the profile from talc-chlorite schist, chlorite-talc schist to talc-chlorite schist. 1 - talc, 2 - chlorite, 3 quartz, 4 - muscovite, 5 - dolomite.
(500 g.t . $\left.^{-1}\right), \mathrm{Na}_{2} \mathrm{CO}_{3}\left(200\right.$ g.t $\left.^{-1}\right)$, water-glass $\left(2,000\right.$ g.t $\left.^{-1}\right)$, $\mathrm{pH}-6$. The tables state also the sampling intervals.

Obtained results confirm that the flotation of talcmagnesite rock type gave a product fulfilling the requirements for pharmaceutical type. In the case of electroceramic technological type the main drawback was the higher content of $\mathrm{Fe}_{2} \mathrm{O}_{3}(1.38 \%)$. Therefore additional dressing using polygradient electromagnetic mud separator was necessary and the $\mathrm{Fe}_{2} \mathrm{O}_{3}$ content was lowered to $1.0-1.16 \%$.

During the flotation of talc from talc-dolomite rock type the natrium hexametaphosphate was used to inhibit dolomite. It depressed dolomite, but lowered the yield of talc product (29.28\%). This product fulfils the criteria for pharmaceutical talc (Table 2).

Dressing of talc-magnesite-dolomite rock type produced talc, which after refining flotation fulfils the norm criteria for electroceramic talc. The talc yield is $50.73 \%$ (Table 3).

Using of refining flotation for the talc-magnesite cell product (Table 4) there was obtained high quality

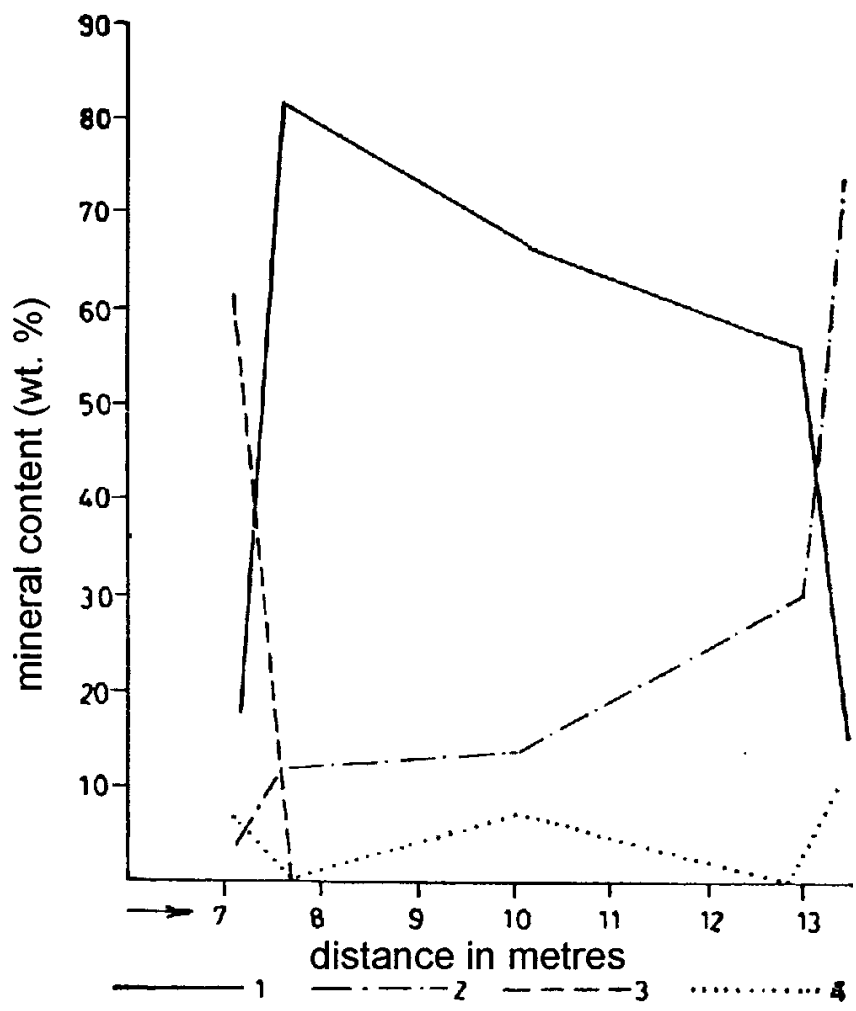

Fig. 3. Distribution of the main rock forming minerals in the deposit in relation to single rock varieties in the profile steatitized magnesite, talc schist, chlorite-talc schist and talc-chlorite schist. 1 - talc, 2 chlorite, 3 - magnesite, 4 - dolomite. 
Table 1: Flotation treatment of talc-magnesite type of raw material

\begin{tabular}{|c|c|c|c|c|c|c|c|c|}
\hline \multirow[b]{2}{*}{$\begin{array}{l}\text { Chemical } \\
\text { and } \\
\text { mineral } \\
\text { composition } \\
\text { (wt. \%) }\end{array}$} & \multicolumn{3}{|c|}{ Primary flotation } & \multicolumn{4}{|c|}{ Refining flotation of talc } & \multirow[b]{2}{*}{$\begin{array}{c}\text { TALC } \\
\text { QUALITY } \\
\text { 00/A } \\
\text { (pharmac. } \\
\text { talc) }\end{array}$} \\
\hline & $\begin{array}{c}\text { Flotation } \\
\text { input }\end{array}$ & $\begin{array}{c}\text { Foam } \\
\text { product } \\
\text { (talc) }\end{array}$ & $\begin{array}{c}\text { Cell } \\
\text { product } \\
\text { (magnesite) }\end{array}$ & $\begin{array}{c}\text { First } \\
\text { sampling } \\
\text { after } 4 \\
\text { minutes }\end{array}$ & $\begin{array}{l}\text { Second } \\
\text { sampling } \\
\text { after } 14 \\
\text { minutes }\end{array}$ & $\begin{array}{c}\text { Foam } \\
\text { product }\end{array}$ & $\begin{array}{c}\text { Cell } \\
\text { product }\end{array}$ & \\
\hline Weight yield (\%) & 100,00 & 10,35 & 89,65 & 4,37 & 2,39 & 6,76 & 3,59 & 100,00 \\
\hline $\mathrm{SiO}_{2}$ & 5,88 & 44,64 & 1,40 & 61,06 & 57,22 & 60,05 & 16,27 & - \\
\hline $\mathrm{Al}_{2} \mathrm{O}_{3}$ & 0,93 & 4,10 & 0,56 & 0,73 & 1,00 & 0,83 & 10,26 & - \\
\hline $\mathrm{Fe}_{2} \mathrm{O}_{3}(\mathrm{t})$ & 1,63 & 1,82 & 1,61 & 1,33 & 1,46 & 1,38 & 2,65 & $\max 2.5$ \\
\hline $\mathrm{MgO}$ & 44,08 & 33,60 & 45,30 & 31,16 & 33,20 & 31,88 & 36,86 & - \\
\hline $\mathrm{CaO}$ & 0,95 & 0,45 & 1,01 & 0,28 & 0,35 & 0,30 & 0,72 & - \\
\hline $\mathrm{Na}_{2} \mathrm{O}$ & 0,03 & 0,03 & 0,03 & 0,03 & 0,03 & 0,03 & 0,04 & - \\
\hline $\mathrm{K}_{2} \mathrm{O}$ & 0,02 & 0,02 & 0,02 & 0,02 & 0,02 & 0,02 & 0,02 & - \\
\hline loss by ignition & 46,25 & 14,82 & 49,88 & 4,99 & 6,31 & 5,40 & 32,57 & $\max 10$ \\
\hline talc & 7,50 & 63,10 & 1,10 & 96,00 & 89,50 & 93,70 & 5,50 & - \\
\hline chlorite & 4,10 & 18,00 & 2,50 & 3,20 & 4,40 & 3,60 & 45,10 & - \\
\hline magnesite & 85,30 & 17,50 & 93,10 & - & 5,10 & 1,80 & 47,00 & - \\
\hline dolomite & 3,10 & 1,40 & 3,30 & 0,80 & 1,00 & 0,90 & 2,40 & - \\
\hline
\end{tabular}

Table 2: Flotation treatment of talc-dolomite type of raw material

\begin{tabular}{|c|c|c|c|c|c|c|c|}
\hline \multirow[b]{2}{*}{$\begin{array}{l}\text { Chemical } \\
\text { and } \\
\text { mineral } \\
\text { composition } \\
\text { (wt. \%) }\end{array}$} & \multirow[b]{2}{*}{$\begin{array}{l}\text { Flotation } \\
\text { input }\end{array}$} & \multicolumn{5}{|c|}{ Refining flotation of talc } & \multirow[b]{2}{*}{$\begin{array}{c}\text { TALC } \\
\text { QUALITY } \\
\text { 00/A } \\
\text { (pharmac. } \\
\text { talc) }\end{array}$} \\
\hline & & $\begin{array}{c}\text { First } \\
\text { sampling } \\
\text { after } 5 \\
\text { minutes }\end{array}$ & $\begin{array}{c}\text { Second } \\
\text { sampling } \\
\text { after } 13 \\
\text { minutes }\end{array}$ & $\begin{array}{c}\text { Third } \\
\text { sampling } \\
\text { after } 20 \\
\text { minutes }\end{array}$ & $\begin{array}{l}\text { Foam } \\
\text { product }\end{array}$ & $\begin{array}{c}\text { Cell } \\
\text { product }\end{array}$ & \\
\hline Weight yield $(\%)$ & 100,00 & 15,82 & 8,21 & 5,25 & 29,28 & 7,08 & 100,00 \\
\hline $\mathrm{SiO}_{2}$ & 33,63 & 59,44 & 59,72 & 60,39 & 59,69 & 39,59 & - \\
\hline $\mathrm{Al}_{2} \mathrm{O}_{3}$ & 1,02 & 0,46 & 0,49 & 0,46 & 0,47 & 1,00 & - \\
\hline $\mathrm{Fe}_{2} \mathrm{O}_{3}(\mathrm{t})$ & 1,79 & 1,48 & 1,67 & 1,46 & 1,53 & 1,82 & $\max 2.5$ \\
\hline $\mathrm{MgO}$ & 26,30 & 30,53 & 29,97 & 29,49 & 29,64 & 27,88 & - \\
\hline $\mathrm{CaO}$ & 12,75 & 1,46 & 1,60 & 1,78 & 1,56 & 9,40 & - \\
\hline $\mathrm{Na}_{2} \mathrm{O}$ & 0,02 & 0,01 & 0,02 & 0,02 & 0,01 & 0,03 & - \\
\hline $\mathrm{K}_{2} \mathrm{O}$ & 0,02 & 0,02 & 0,02 & 0,02 & 0,02 & 0,02 & - \\
\hline loss by ignition & 24,09 & 6,29 & 6,30 & 6,21 & 6,28 & 19,89 & $\max 10$ \\
\hline talc & 51,00 & 93,20 & 92,60 & 92,30 & 92,90 & 62,60 & - \\
\hline chlorite & 4,50 & 2,00 & 2,20 & 2,00 & 2,10 & 4,50 & - \\
\hline dolomite & 44,50 & 4,80 & 5,20 & 5,70 & 5,00 & 32,90 & - \\
\hline
\end{tabular}


Table 3: Flotation treatment of talc-magnesite-dolomite type of raw material

\begin{tabular}{|c|c|c|c|c|c|c|}
\hline \multirow[b]{2}{*}{$\begin{array}{l}\text { Chemical } \\
\text { and } \\
\text { mineral } \\
\text { composition } \\
\text { (wt. \%) }\end{array}$} & \multirow[b]{2}{*}{$\begin{array}{c}\text { Flotation } \\
\text { input }\end{array}$} & \multicolumn{4}{|c|}{ Refining flotation of talc } & \multirow[b]{2}{*}{$\begin{array}{c}\text { TALC } \\
\text { QUALITY } \\
\text { EK-I } \\
\text { (electrotech. } \\
\text { talc) } \\
\end{array}$} \\
\hline & & $\begin{array}{c}\text { First } \\
\text { sampling } \\
\text { after } 5 \\
\text { minutes }\end{array}$ & $\begin{array}{c}\text { Second } \\
\text { sampling } \\
\text { after } 10 \\
\text { minutes }\end{array}$ & $\begin{array}{l}\text { Foam } \\
\text { product }\end{array}$ & $\begin{array}{l}\text { Cell } \\
\text { product }\end{array}$ & \\
\hline Weight yield (\%) & 100,00 & 36,60 & 14,13 & 50,73 & 9,67 & 100,00 \\
\hline $\mathrm{SiO}_{2}$ & 40,85 & 60,92 & 63,55 & 61,65 & 46,58 & \\
\hline $\mathrm{Al}_{2} \mathrm{O}_{3}$ & 0,66 & 0,26 & 0,22 & 0,25 & 0,96 & $\max 5.0$ \\
\hline $\mathrm{Fe}_{2} \mathrm{O}_{3}(\mathrm{t})$ & 0,82 & 0,55 & 0,57 & 0,56 & 0,74 & $\max 0.7$ \\
\hline $\mathrm{MgO}$ & 31,93 & 31,76 & 31,56 & 31,70 & 31,03 & $\min 28.0$ \\
\hline $\mathrm{CaO}$ & 5,41 & 0,80 & 0,60 & 0,75 & 3,94 & - \\
\hline $\mathrm{Na}_{2} \mathrm{O}$ & 0,02 & 0,02 & 0,02 & 0,02 & 0,02 & - \\
\hline $\mathrm{K}_{2} \mathrm{O}$ & 0,02 & 0,02 & 0,02 & 0,02 & 0,03 & - \\
\hline loss by ignition & 19,85 & 5,24 & 5,27 & 4,75 & 17,15 & $\max 6.0$ \\
\hline talc & 63,40 & 96,60 & 97,00 & 96,70 & 75,50 & - \\
\hline chlorite & 2,80 & 1,10 & 1,00 & 1,10 & 2,50 & - \\
\hline magnesite & 17,50 & - & - & - & 9,00 & \\
\hline dolomite & 16,30 & 2,30 & 2,00 & 2,20 & 13,00 & - \\
\hline
\end{tabular}

Table 4: Flotation treatment of talc-magnesite type of raw material

\begin{tabular}{|c|c|cc|cc|c|}
\hline $\begin{array}{c}\text { Chemical } \\
\text { and } \\
\text { mineral } \\
\text { composition } \\
\text { (wt. \%) }\end{array}$ & $\begin{array}{c}\text { Cell } \\
\text { product } \\
\text { (input) }\end{array}$ & $\begin{array}{c}\text { Primary flotation } \\
\text { Foam }\end{array}$ & $\begin{array}{c}\text { Cell } \\
\text { product }\end{array}$ & $\begin{array}{c}\text { Refining flotation } \\
\text { product }\end{array}$ & $\begin{array}{c}\text { TALC } \\
\text { product } \\
\text { QUALITY } \\
\text { la - Ib } \\
\text { (rubbery } \\
\text { industry) }\end{array}$ \\
\hline Weight yield (\%) & 89,65 & 64,48 & 25,17 & 56,23 & 8,25 & 100,00 \\
\hline $\mathrm{SiO}_{2}$ & 1,40 & 0,95 & 2,57 & 0,51 & 3,95 & $\operatorname{max~} 1.5$ \\
$\mathrm{Al}_{2} \mathrm{O}_{3}$ & 0,56 & 0,34 & 1,13 & 0,14 & 1,74 & - \\
$\mathrm{Fe}_{2} \mathrm{O}_{3}(\mathrm{t})$ & 1,61 & 1,56 & 1,71 & 1,56 & 1,51 & $\max 2.42$ \\
$\mathrm{MgO}_{\mathrm{CaO}}$ & 45,30 & 45,78 & 44,10 & 46,09 & 43,66 & $\min 44.4$ \\
$\mathrm{Na}_{2} \mathrm{O}$ & 1,01 & 0,94 & 1,18 & 0,92 & 1,08 & $\max 0.90$ \\
$\mathrm{~K}_{2} \mathrm{O}$ & 0,03 & 0,03 & 0,03 & 0,02 & 0,04 & - \\
loss by ignition & 0,02 & 0,02 & 0,02 & 0,02 & 0,02 & - \\
\hline talc & 49,88 & 50,26 & 48,90 & 50,65 & 47,59 & - \\
chlorite & 1,10 & 0,80 & 1,90 & 0,50 & 2,80 & - \\
magnesite & 93,50 & 1,50 & 5,10 & 0,60 & 7,80 & - \\
dolomite & 3,30 & 94,60 & 89,10 & 95,90 & 85,90 & - \\
\hline
\end{tabular}


magnesite product. The yield of magnesite is $56.23 \%$. This type magnesite was successfully tested as an additive for wall-plaster substances.

Beside the yield of talc, the primary flotation of talcmagnesite raw material also allows to gain magnesite concentrate of technological type usable in rubbery industry with $89.65 \%$ yield of magnesite concentrate (Table 4 ).

\section{Talcose rocks from Kokava deposit}

Three talcose rock types were studied from the viewpoint of flake industrial use:

The first type is represented by sericite-chlorite schist with predominance of tabular mineral grains in foliation. The orientation of tabular minerals is either parallel or irregular. The chemical and mineral compositions are displayed in Table 5.

The second rock type, steatitized sericite-chlorite schist, and third rock type, strongly steatitized chlorite schist, consist of combination of mosaic of equidimensional and tabular minerals of irregular orientation.

The evaluation of the quality of obtained products was made using quantitative shape analysis of particles. Grains have been assigned as flakes in the cases where the elongation/thickness ratio was $3: 1$ or higher. Qualitative analysis of flakes was made under microscope in thin section (Table 5), as well as by chemical and X-ray analysis (Derco \& Vlasák 1984; Table 6).The relation of weight yield to granularity was studied in the case of the first rock type (sericite-chlorite schist; Fig. 4).

Table 5: Partial chemical composition, calculated mineralogical composition and average flake content in principal samples of three investigated rock types.

\begin{tabular}{|c|c|c|c|c|c|c|c|c|c|c|c|c|c|}
\hline $\begin{array}{l}\text { Rock } \\
\text { type }\end{array}$ & $\begin{array}{l}\text { Sample } \\
\text { number }\end{array}$ & $\begin{array}{c}\text { Average } \\
\text { flakeness } \\
\%\end{array}$ & $\mathrm{SiO}_{2}$ & $\begin{array}{l}\text { Partia } \\
\mathrm{Al}_{2} \mathrm{O}_{3}\end{array}$ & $\begin{array}{c}\text { hemical } \\
\text { wt. \% } \\
\text { MgO }\end{array}$ & $\begin{array}{l}\text { Partial chemical analysis } \\
\text { wt. \% }\end{array}$ & \multicolumn{2}{|l|}{$\mathrm{Al}_{2} \mathrm{O}_{3}+$} & \multicolumn{4}{|c|}{ Mineral composition (wt. \%) } & $\begin{array}{l}\text { chlorite+ } \\
\text { sericite+ } \\
\text { talc }\end{array}$ \\
\hline \multirow{5}{*}{$\begin{array}{c}\text { Type } 1 \\
\text { Sericite-chlorite } \\
\text { schist }\end{array}$} & 3 & 27,16 & 59,93 & 15,93 & 9,74 & 2,11 & 27,78 & 2,16 & 38,58 & 21,10 & - & 40,32 & 59,62 \\
\hline & 5 & 11,85 & 70,91 & 10,74 & 7,42 & 1,37 & 19,53 & 3,63 & 26,84 & 13,70 & - & 59,46 & 40,54 \\
\hline & 6 & 22,55 & 67,27 & 13,74 & 7,65 & 2,17 & 23,56 & 2,86 & 27,86 & 21,70 & - & 50,44 & 49,56 \\
\hline & 17 & 18,15 & 70,81 & 13,18 & 5,60 & 2,50 & 21,28 & 3,33 & 20,27 & 25,00 & - & 54,73 & 45,27 \\
\hline & 18 & 24,03 & 66,88 & 14,23 & 7,70 & 2,35 & 24,28 & 2,75 & 27,28 & 23,50 & - & 49,22 & 50,78 \\
\hline \multirow{6}{*}{$\begin{array}{c}\text { Type } 2 \\
\text { Steatitized } \\
\text { sericite-chlorite } \\
\text { schist }\end{array}$} & 7 & 19,76 & 62,97 & 10,49 & 12,17 & 0,66 & 23,32 & 2,70 & 36,66 & 6,60 & 7,37 & 49,37 & 50,63 \\
\hline & 9 & 45,88 & 59,13 & 12,40 & 16,93 & 0,68 & 30,01 & 1,97 & 44,88 & 6,80 & 15,58 & 34,03 & 67,26 \\
\hline & 12 & \multirow{2}{*}{31,87} & 60,05 & 13,43 & 12,91 & 1,01 & 27,35 & 2,20 & 44,39 & 10,10 & 3,00 & 42,51 & 57,40 \\
\hline & 13 & & 60,08 & 12,79 & 13,39 & 0,81 & 26,99 & 2,23 & 44,61 & 8,10 & 4,36 & 42,93 & 57,07 \\
\hline & 14 & \multirow{2}{*}{37,67} & 58,96 & 11,86 & 17,84 & 0,02 & 29,72 & 1,98 & 52,70 & 0,20 & 11,70 & 35,40 & 64,60 \\
\hline & 15 & & 56,17 & 12,94 & 18,62 & 0,08 & 31,61 & 1,78 & 56,58 & 0,80 & 10,83 & 31,79 & 68,21 \\
\hline \multirow{2}{*}{$\begin{array}{c}\text { Type } 3 \text { Strongly } \\
\text { steatitiz.chlorite schist }\end{array}$} & 8 & \multirow{2}{*}{24,37} & 53,14 & 9,82 & 22,67 & 0,19 & 32,68 & 1,63 & 40,95 & 1,90 & 37,56 & 19,59 & 80,41 \\
\hline & 16 & & 37,49 & 17,84 & 27,71 & 0,02 & 45,57 & 0,82 & 79,41 & 0,20 & 20,27 & 0,12 & 99,88 \\
\hline
\end{tabular}

Table 6: X-ray diffraction records of principal samples representing individual rock types.

\begin{tabular}{|c|c|c|c|c|c|c|c|c|c|c|c|c|c|}
\hline \multicolumn{6}{|c|}{ Measured values in $\mathrm{nm}$} & \multicolumn{8}{|c|}{ Table values in $\mathrm{nm}$} \\
\hline \multicolumn{2}{|c|}{$\begin{array}{l}\text { Type } 1 \\
\text { Sample } \\
\text { No. } 5 \\
\end{array}$} & \multicolumn{2}{|c|}{$\begin{array}{c}\text { Type } 2 \\
\text { Sample } \\
\text { No. } 9 \\
\end{array}$} & \multicolumn{2}{|c|}{$\begin{array}{c}\text { Type } 3 \\
\text { Sample } \\
\text { No. } 8 \\
\end{array}$} & \multicolumn{2}{|c|}{ chlorite } & \multicolumn{2}{|c|}{$\begin{array}{l}\text { muscovite } \\
\text { (sericite) }\end{array}$} & \multicolumn{2}{|c|}{ talc } & \multicolumn{2}{|c|}{ quartz } \\
\hline d & 1 & d & 1 & d & 1 & d & 1 & $\mathrm{~d}$ & $\mathrm{I}$ & d & 1 & d & 1 \\
\hline 1,410 & 6 & 1,410 & 6 & 1,410 & 7 & 1,410 & 7 & - & & - & & - & \\
\hline 0,992 & 3 & 0,992 & 1 & - & & - & & 0,999 & 95 & - & & - & \\
\hline- & & 0,930 & 1 & 0,930 & 9 & - & & - & & 0,930 & & - & \\
\hline 0,708 & 10 & 0,709 & 10 & 0,710 & 10 & 0,707 & 9 & - & & - & & - & \\
\hline 0,497 & 1 & - & & - & & - & & 0,498 & 30 & - & & - & \\
\hline 0,472 & 6 & 0,473 & 7 & 0,473 & 7 & 0,472 & 8 & - & & - & & - & \\
\hline 0,425 & 2 & 0,425 & 1 & 0,425 & 0,5 & 0,354 & 10 & - & & - & & 0,426 & 35 \\
\hline 0,353 & 9 & 0,353 & 9 & 0,354 & 9 & - & & - & & - & & - & \\
\hline 0,334 & 9 & 0,334 & 5 & 0,333 & 3 & - & & 0,332 & 100 & - & & 0,334 & 100 \\
\hline- & & 0,311 & 1 & 0,311 & 10 & - & & - & & 0,310 & 70 & - & \\
\hline 0,284 & 2 & - & & - & & 0,284 & 5 & - & & - & & - & \\
\hline
\end{tabular}




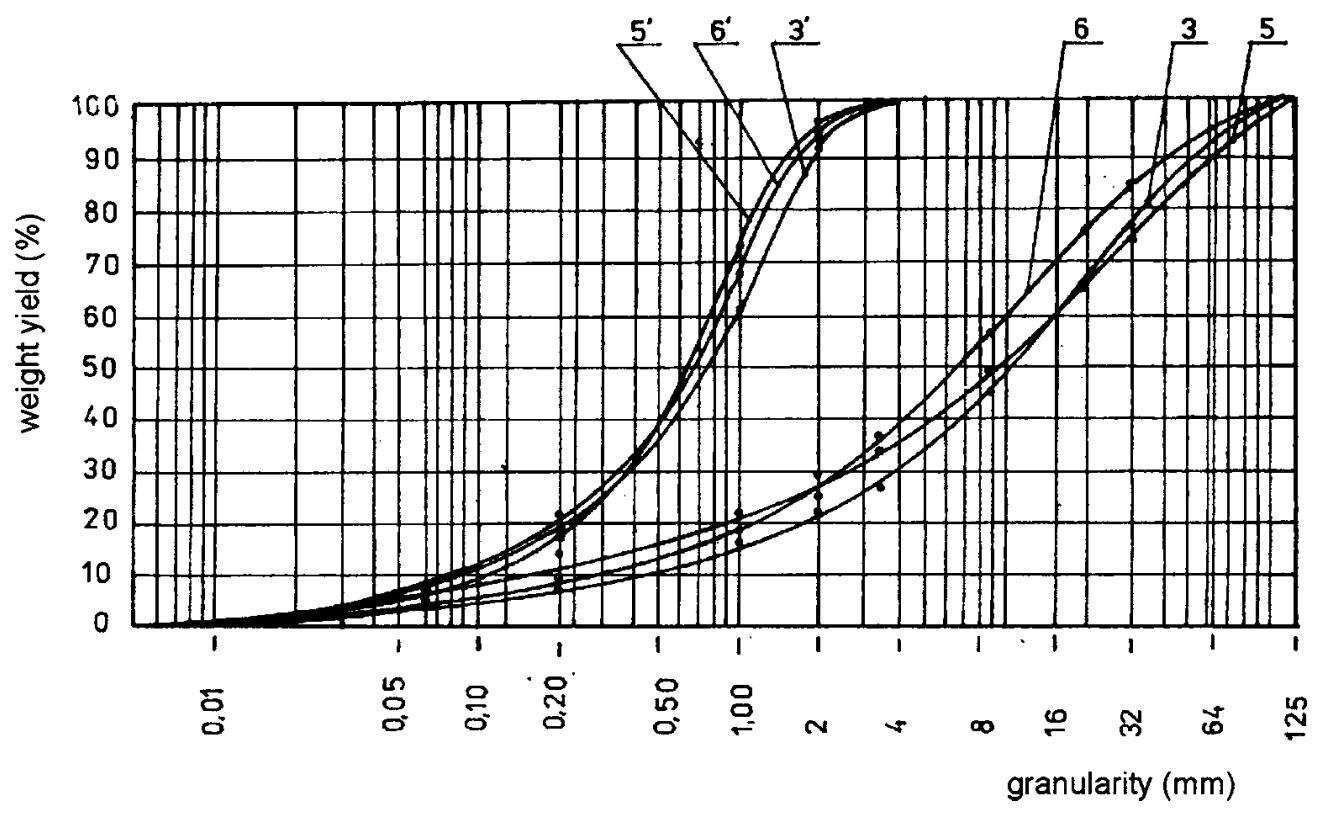

Fig. 4. Granularity curves of the first rock type samples (sericite-chlorite schist, samples 3, 5, 6) expressed in the polarographic net. 3, 5, 6 - primary samples after $1^{\text {st }}$ grinding, $3^{\circ}, 5^{\circ}, 6^{\text {c }}$ - the same samples after $2^{\text {nd }}$ grinding.

\section{Technological properties of the raw material}

The specific gravity of rock fluctuates between 2.70 and $2.74{\mathrm{~g} . \mathrm{cm}^{-3}}^{-3}$. In the course of primary crushing of samples with higher moisture (over 5 wt. \%) problems arised during sizing due to its adhesive power. Considerable amounts of dust particles (under $0.1 \mathrm{~mm}$ size) originated during the drying of the crushed material.

\section{Characteristics of flakes and granules}

Flakes from sericite-chlorite schist reveal the following mineral composition: chlorite $(47 \%)$, sericite (28\%) and quartz (25\%). The share of flakes in the sample is fluctuating between 11 and 27 wt. \%. The granules consist of quartz (55-58 \%), chlorite (28\%) and sericite $(17 \%)$ and their amount fluctuates between 73 and $89 \%$ (Tab. 5)

Flakes of the steatitized sericite-chlorite schist consist of chlorite (75\%), quartz (10\%), sericite (8\%) and talc $(7 \%)$, the amount of flakes is $19-46 \mathrm{wt} . \%$. The rest of the rock represent quartz (55\%), chlorite (36 $\%)$, sericite $(6 \%)$ and talc $(2.8 \%)$.

Mineral composition of the flakes of the strongly steatitized chlorite schist consists of chlorite (67\%), talc $(17.5 \%)$, quartz $(9.5 \%)$ and sericite $(6 \%)$. The average content of flakes is roughly $24 \%$ and the rest of rock consists of quartz (34\%), chlorite (36\%), talc $(29 \%)$ and sericite $(1 \%)$ and the average yield of granule is around $76 \%$ (Tab. 5).

As follows from the previous, the second rock type, the steatitized sericite-chlorite schist, is the most suitable for flake production. The flake material has low quartz contents and the best shape indices. Unsuitable for the flake production is the third rock type because the content of flakes is low. The high talc content assigns this type prevailingly as raw material for the rubber industry.

\section{Relations between the amount of flakes, mineralogy and chemical composition}

According to the analytical results, higher content of quartz in the rock results in lower amounts of flakes whereas higher chlorite and sericite content is reflected by higher flake production. Steatitizations beneficates the flake content only in amounts up to 10-15\%. This finding has been proved on artificial samples of the 0.56 $1.00 \mathrm{~mm}$ size fraction representing the composition of average primary sample. The flake content in sample prepared was $0-50-100 \%$ as indicated in Table 7 and Figs. 5, 6 and 7. 
DERCO, J.; NÉMETH, Z. Obtaining of high quality talc from talcose rocks: A case study from the Sinec and Kokava deposits (Slovakia)

Table 7: Partial chemical composition and calculated mineralogical composition of artificial samples with 0-50-100 \% of flakes from the $0.56-1.00 \mathrm{~mm}$ size fraction.

\begin{tabular}{|c|c|c|c|c|c|c|c|c|c|c|c|c|}
\hline \multirow{2}{*}{$\begin{array}{l}\text { Rock } \\
\text { type }\end{array}$} & \multirow{2}{*}{$\begin{array}{c}\text { Flake } \\
\text { content } \\
(\%)\end{array}$} & \multicolumn{6}{|c|}{$\begin{array}{l}\text { Partial chemical composition } \\
\text { (wt. \%) }\end{array}$} & \multicolumn{5}{|c|}{$\begin{array}{l}\text { Mineral composition } \\
\text { (wt. \%) }\end{array}$} \\
\hline & & $\mathrm{SiO}_{2}$ & $\mathrm{Al}_{2} \mathrm{O}_{3}$ & $\mathrm{MgO}$ & $\mathrm{K}_{2} \mathrm{O}$ & $\begin{array}{c}\mathrm{Al}_{2} \mathrm{O}_{3}+ \\
\mathrm{MgO}+ \\
\mathrm{K}_{2} \mathrm{O}\end{array}$ & $\mathrm{K}+$ & chlorite & sericite & talc & quartz & $\begin{array}{c}\text { chlor.+ } \\
\text { seric. }+ \\
\text { talc }\end{array}$ \\
\hline \multirow{3}{*}{ Type 1} & 0 & 72,07 & 12,25 & 6,19 & 1,70 & 20,14 & 3,58 & 28,45 & 17,00 & - & 54,55 & 45,45 \\
\hline & 50 & 59,80 & 15,24 & 11,31 & 1,40 & 27,95 & 2,14 & 46,44 & 14,00 & - & 39,56 & 60,44 \\
\hline & 100 & 50,89 & 21,26 & 11,40 & 2,80 & 35,46 & 1,44 & 47,50 & 28,00 & - & 24,50 & 75,50 \\
\hline \multirow{3}{*}{ Type 2} & 0 & 65,94 & 10,06 & 10,67 & 0,56 & 21,29 & 3,10 & 36,31 & 5,60 & 2,81 & 55,28 & 44,72 \\
\hline & 50 & 51,61 & 14,94 & 18,19 & 0,58 & 33,71 & 1,53 & 57,79 & 5,80 & 8,37 & 28,04 & 71,96 \\
\hline & 100 & 41,66 & 19,43 & 22,29 & 0,79 & 42,51 & 0,98 & 74,58 & 7,90 & 6,98 & 10,54 & 89,46 \\
\hline \multirow{3}{*}{ Type 3} & 0 & 56,81 & 8,66 & 18,70 & 0,15 & 27,51 & 2,07 & 36,40 & 1,50 & 28,70 & 33,40 & 66,60 \\
\hline & 50 & 49,98 & 14,29 & 22,02 & 0,36 & 36,67 & 1,36 & 58,28 & 3,60 & 20,93 & 17,19 & 82,81 \\
\hline & 100 & 44,52 & 17,04 & 23,58 & 0,57 & 41,19 & 1,08 & 67,30 & 5,70 & 17,49 & 9,51 & 90,49 \\
\hline
\end{tabular}

$\mathrm{K}+=\mathrm{SiO}_{2} /\left(\mathrm{Al}_{2} \mathrm{O}_{3}+\mathrm{MgO}+\mathrm{K}_{2} \mathrm{O}\right)$

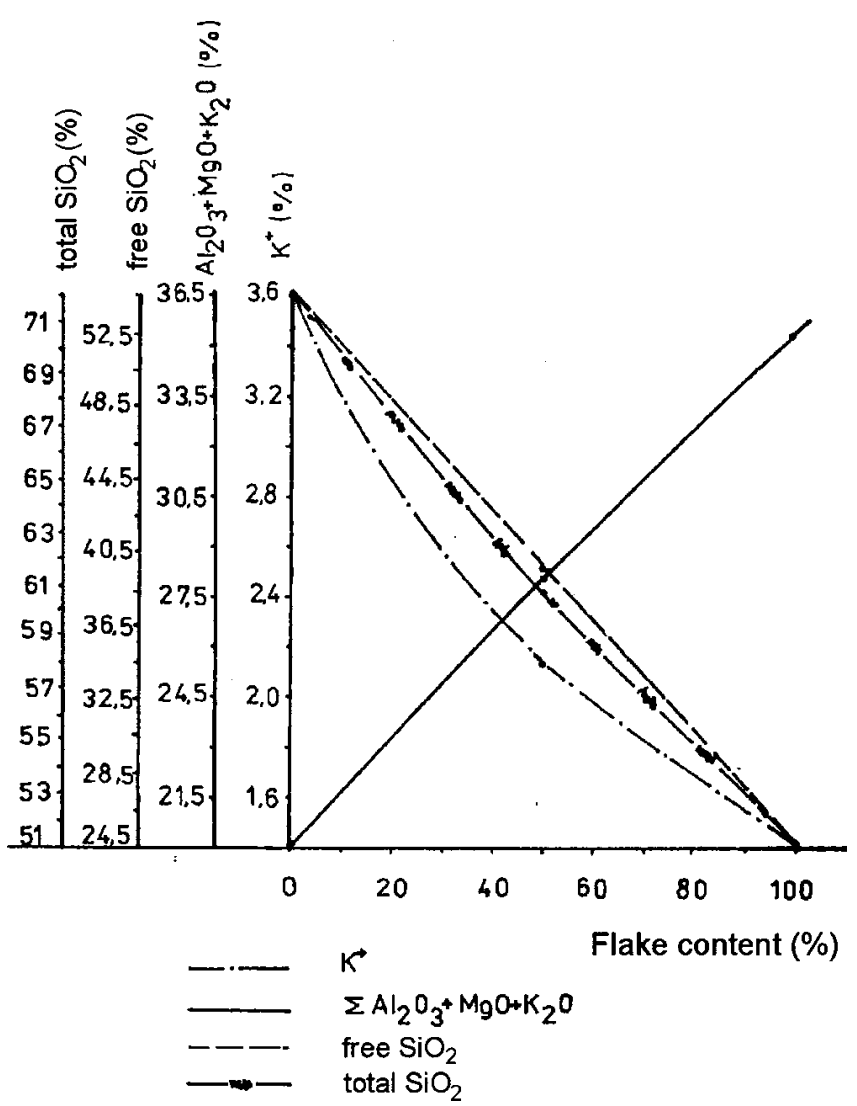

Fig. 5. Relation between the flake content and $\mathrm{K}+$ coefficient, total $\mathrm{SiO}_{2}$, free $\mathrm{SiO}_{2}, \mathrm{Al}_{2} \mathrm{O}_{3}+\mathrm{MgO}+\mathrm{K}_{2} \mathrm{O}$ of the first rock type (sericitechlorite schist; $0.56-1.00 \mathrm{~mm}$ size fraction).

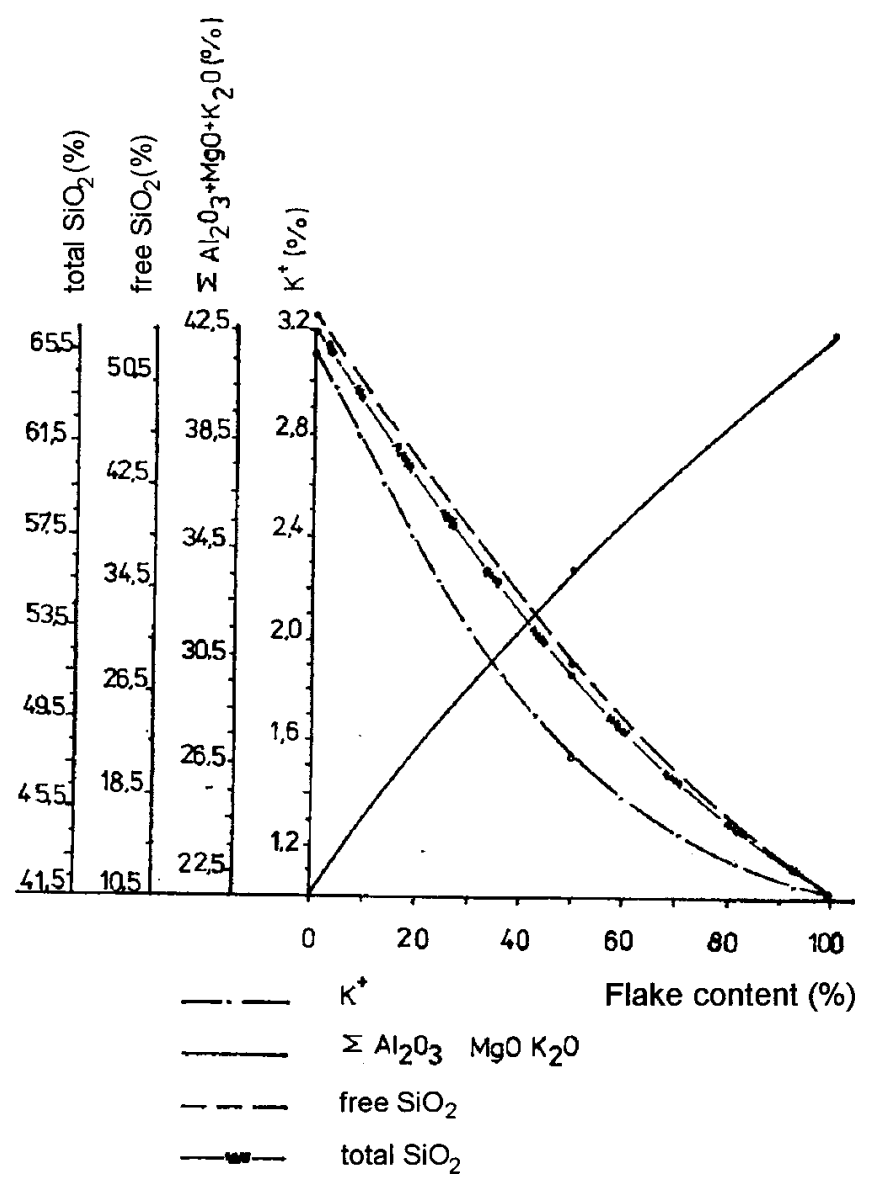

Fig. 6. Relation between the flake content and $\mathrm{K}+$ coefficient, total $\mathrm{SiO}_{2}$, free $\mathrm{SiO}_{2}, \mathrm{Al}_{2} \mathrm{O}_{3}+\mathrm{MgO}+\mathrm{K}_{2} \mathrm{O}$ of the second rock type (steatitized sericite-chlorite schist; $0.56-1.00 \mathrm{~mm}$ size fraction). 


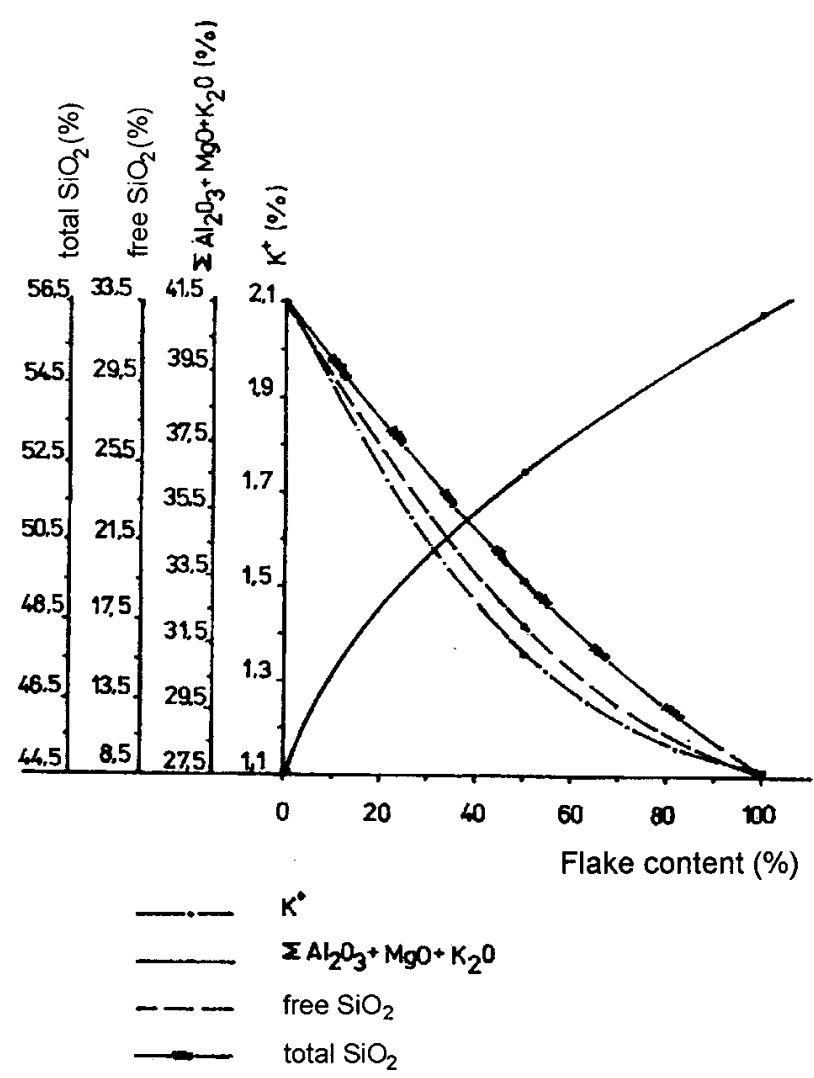

Fig. 7. Relation between the flake content and $\mathrm{K}+$ coefficient, total $\mathrm{SiO}_{2}$, free $\mathrm{SiO}_{2}, \mathrm{Al}_{2} \mathrm{O}_{3}+\mathrm{MgO}+\mathrm{K}_{2} \mathrm{O}$ of the third rock type (strongly steatitized chlorite schist; $0.56-1.00 \mathrm{~mm}$ size fraction).

\section{Relation between the flake content of prepared products and bulk weight}

The higher is the share of flaky grains in the single preparation products the greater is their volume in the case of loose pouring. Hence the bulk weight may be used to control the amount of flakes produced. For the sake of reproduceability, the routing indicated schematically in Fig. 6 has been applied. Results are in Tab. 8 .

Because the weight of a loosely poured material was necessary to measure, the important size of the 5 slot has been designed $5 \times 50 \mathrm{~mm}$ and the grain filling height into the calibrated vessel was $200 \mathrm{~mm}$.

For the assessment of the relation between the bulk weight and the flake content, the artificial samples containing various amounts of flakes have been prepared. It was proved that the relation between the bulk weight and amount of flakes may be expressed by an exponential function of

$$
\beta=\mathrm{k} \cdot \mathrm{z}^{\mathrm{n}}
$$

where $\beta=$ maximal - expected flake content in wt. $\%, z$ $=0-1$, where 0 means minimal and 1 maximal bulk weight, $\mathrm{k}=$ maximal-minimal flake content.

The comparison of curves obtained from artificial samples with the calculated values is in Fig. 8. The

Table 8: Relation between bulk weight and flake content

\begin{tabular}{|c|c|c|c|c|c|c|c|}
\hline \multirow{3}{*}{$\begin{array}{l}\text { Rock } \\
\text { type }\end{array}$} & \multirow{3}{*}{$\begin{array}{l}\text { Sample } \\
\text { type }\end{array}$} & \multicolumn{6}{|c|}{ Grain size $(\mathrm{mm})$} \\
\hline & & \multicolumn{2}{|c|}{$1 \mathrm{~mm}-2 \mathrm{~mm}$} & \multicolumn{2}{|c|}{$0.56 \mathrm{~mm}-1 \mathrm{~mm}$} & \multicolumn{2}{|c|}{$0.2 \mathrm{~mm}-0.56 \mathrm{~mm}$} \\
\hline & & $\begin{array}{c}\text { Bulk } \\
\text { weight } \\
\left(\text { g. }\left.\right|^{-1}\right)\end{array}$ & $\begin{array}{c}\text { Flake } \\
\text { content } \\
(\%)\end{array}$ & $\begin{array}{c}\text { Bulk } \\
\text { weight } \\
\left(\text { g. } \text { I }^{-1}\right)\end{array}$ & $\begin{array}{c}\text { Flake } \\
\text { content } \\
(\%)\end{array}$ & $\begin{array}{c}\text { Bulk } \\
\text { weight } \\
\left(\mathrm{g} . \mathrm{I}^{-1}\right)\end{array}$ & $\begin{array}{c}\text { Flake } \\
\text { content } \\
(\%)\end{array}$ \\
\hline \multirow{10}{*}{ Type 1} & $\mathrm{~T}$ & 1160 & 37,00 & 1087 & 38,24 & 957 & 39,10 \\
\hline & $\mathrm{T}$ & 1180 & 27,79 & 1140 & 20,63 & 1020 & 20,15 \\
\hline & $\mathrm{N}$ & 1182 & 26,20 & 1104 & 29,36 & 975 & 31,61 \\
\hline & $\mathrm{T}$ & 1187 & 26,00 & 1122 & 23,50 & 970 & 34,13 \\
\hline & $\mathrm{N}$ & 1203 & 20,35 & 1125 & 24,33 & 984 & 26,25 \\
\hline & $\mathrm{N}$ & 1208 & 18,55 & 1150 & 20,18 & 1018 & 20,90 \\
\hline & $\mathrm{N}$ & 1219 & 14,56 & 1190 & 13,84 & 1049 & 17,50 \\
\hline & $\mathrm{N}$ & 1225 & 14,00 & 1178 & 15,35 & 1028 & 19,42 \\
\hline & $\mathrm{N}$ & 1235 & 13,50 & 1183 & 15,15 & 1031 & 18,87 \\
\hline & $\mathrm{T}$ & 1240 & 11,50 & 1296 & 3,37 & 1258 & 6,53 \\
\hline \multirow{4}{*}{ Type 2} & $\mathrm{~N}$ & 1030 & 43,40 & 975 & 44,19 & 857 & 55,60 \\
\hline & $\mathrm{N}$ & 1072 & 35,15 & 1010 & 37,90 & 893 & 40,00 \\
\hline & $\mathrm{N}$ & 1109 & 28,70 & 1039 & 31,54 & 917 & 35,37 \\
\hline & $\mathrm{N}$ & 1239 & 14,19 & 1198 & 19,08 & 1075 & 21,25 \\
\hline Type 3 & $\mathrm{~N}$ & 1199 & 25,60 & 1146 & 24,09 & 1018 & 28,57 \\
\hline
\end{tabular}

$\mathrm{T}$ - artificially prepared technological sample, $\mathrm{N}$ - natural sample 
DERCO, J.; NÉMETH, Z. Obtaining of high quality talc from talcose rocks: A case study from the Sinec and Kokava deposits (Slovakia)

Table 9. Relative flake content and partial chemical composition of weight fraction in the 1-2 mm size class.

\begin{tabular}{|c|c|c|c|c|c|c|c|}
\hline \multirow{2}{*}{$\begin{array}{l}\text { Rock } \\
\text { type }\end{array}$} & \multirow{2}{*}{$\begin{array}{c}\text { Bulk spec. } \\
\text { gravity } \\
\text { (g.cm-3) }\end{array}$} & \multirow{2}{*}{$\begin{array}{c}\text { Flake } \\
\text { content } \\
(\%)\end{array}$} & \multicolumn{5}{|c|}{ Chemical composition (\%) } \\
\hline & & & $\mathrm{SiO}_{2}$ & $\mathrm{Al}_{2} \mathrm{O}_{3}$ & $\mathrm{MgO}$ & $\mathrm{K}_{2} \mathrm{O}$ & $\begin{array}{l}\text { Loss by } \\
\text { ignition }\end{array}$ \\
\hline \multirow{4}{*}{ Type 1} & $-2,67$ & 10,04 & 75,77 & 6,33 & 8,24 & - & 4,04 \\
\hline & $2.67-2.72$ & 16,50 & 72,60 & 10,37 & 7,24 & - & 4,02 \\
\hline & $2.72-2.75$ & 50,03 & 55,89 & 18,67 & 9,65 & - & 5,80 \\
\hline & 2,75 & 45,50 & 58,34 & 15,94 & 8,46 & - & 6,07 \\
\hline \multirow{4}{*}{ Type 2} & $-2,67$ & 17,50 & 54,42 & 13,23 & 20,41 & 0,26 & 7,97 \\
\hline & $2.67-2.72$ & 40,87 & 52,64 & 15,81 & 19,77 & 0,89 & 7,10 \\
\hline & $2.72-2.75$ & 60,13 & 45,52 & 18,90 & 21,41 & 1,54 & 8,61 \\
\hline & 2,75 & 55,22 & 46,76 & 17,38 & 20,69 & 2,30 & 8,42 \\
\hline \multirow{4}{*}{ Type 3} & $-2,67$ & 12,63 & 72,22 & 8,35 & 12,03 & 0,13 & 5,03 \\
\hline & $2.67-2.72$ & 43,00 & 61,80 & 12,09 & 16,17 & 0,41 & 6,91 \\
\hline & $2.72-2.75$ & 31,51 & 44,89 & 15,04 & 8,84 & 0,56 & 9,04 \\
\hline & 2,75 & 12,60 & 38,99 & 6,51 & 28,41 & 0,51 & 16,78 \\
\hline
\end{tabular}

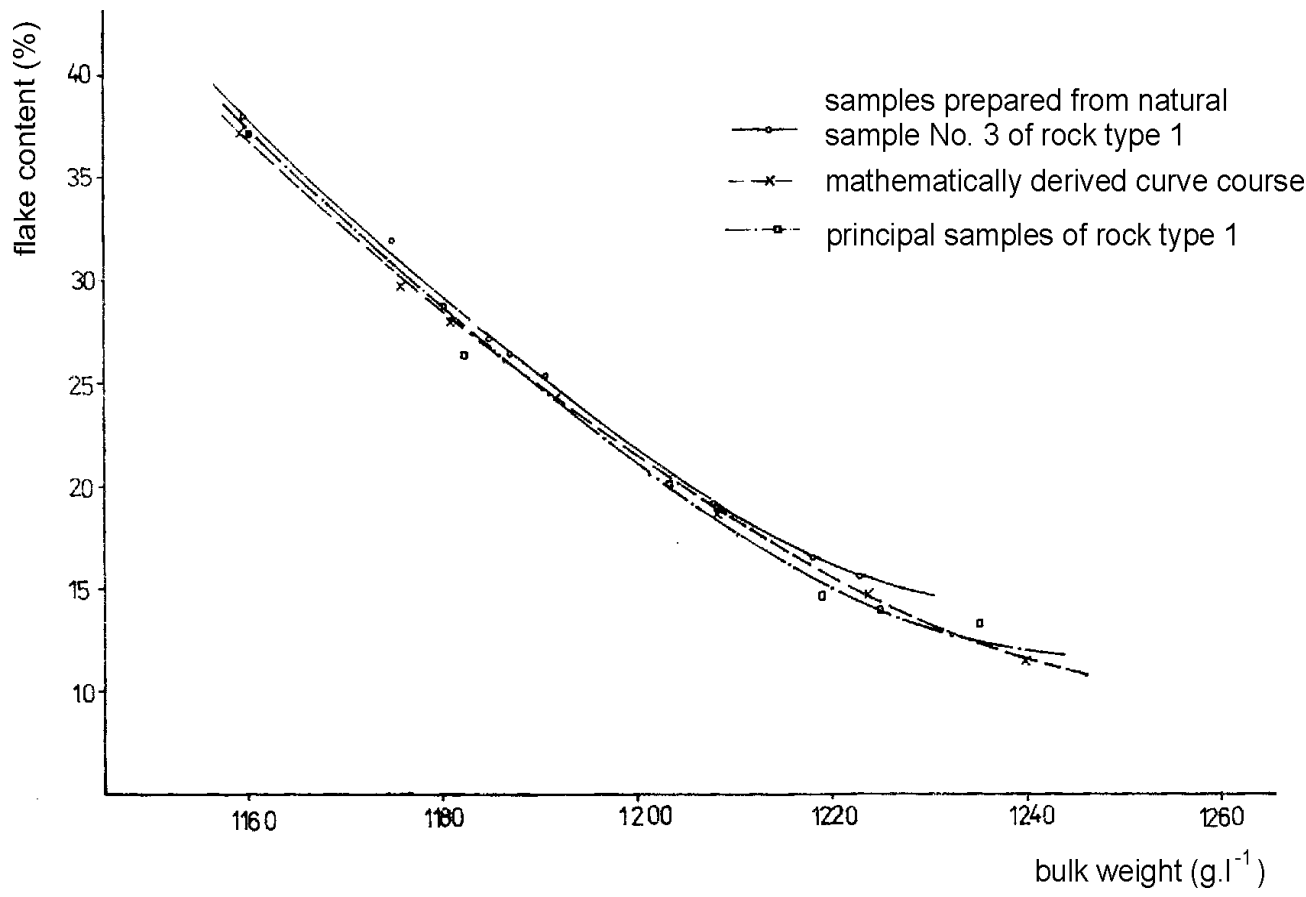

Fig. 8. Relation between the bulk weight and flake content of the first rock type, 1-2 $\mathrm{mm}$ size fraction. 


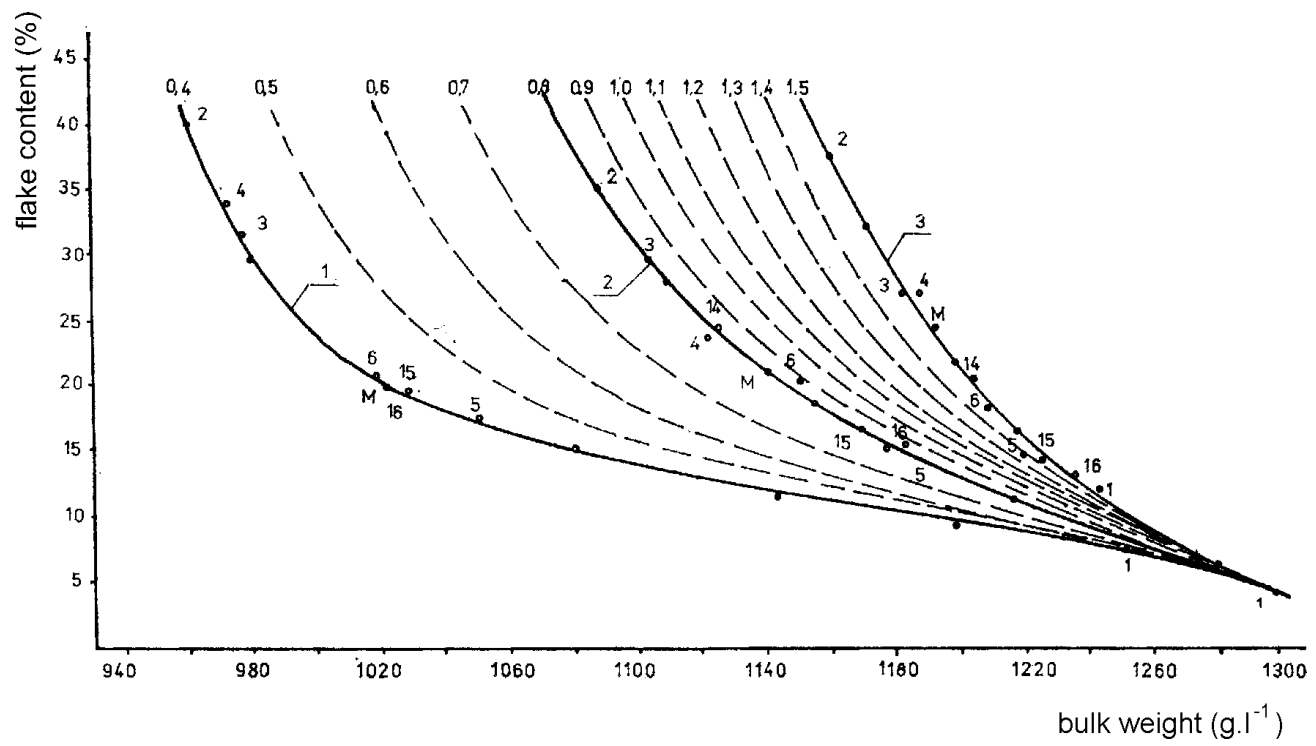

Fig. 9. Relation between bulk weight and flake content of samples $1-0.2-0.56 \mathrm{~mm}$ size fraction, $2-0.56-1.00 \mathrm{~mm}$ size fraction, 3 - 1.00-2.00 size fraction, $\mathrm{M}$ - artificial sample prepared for the calculation of the curve, 3, 5, 6 - principal samples of the first type of rock, 1,2, M, 4 - artificial samples, 14, 15, 16 - further samples of the first type of rock prepared for testing of methodics, ... - points without designation were calculated mathematically.

relation between bulk weight and flake content of samples of individual grain size fractions of the first rock type is demonstrated in Fig. 9.

The information about the change of flakeness and chemical composition of products in relation to their bulk specific gravity was obtained using heavy fluid separation (tetrabrometane; Tab. 9). The decrease of amount of flake material in weight fractions as well as increase of the number of granules in the third rock type above value $2.73 \mathrm{~g} . \mathrm{cm}^{-3}$ was caused by presence of carbonates (dolomite).

\section{Laboratory tests of further upgrading}

Obtained flaky products may be further upgraded with the aim to increase the flake content and to suppress the amount of dust particles under $0.1 \mathrm{~mm}$ size.

Upgrading tests were performed using the following laboratory devices

- fixed slot grate

- concentrating table operating moisture-free, rubber lined

- electromagnetic vibration screen operating with triboadhesion effect

Table 10: Results obtained during upgrading of grinding and sizing products.

\begin{tabular}{|c|c|c|c|c|c|c|c|}
\hline \multirow[b]{2}{*}{ Used devices } & \multicolumn{3}{|c|}{ Inputted raw material } & \multicolumn{4}{|c|}{ Assorting after additional upgrading } \\
\hline & $\begin{array}{l}\text { Grain size } \\
(\mathrm{mm})\end{array}$ & $\begin{array}{c}\text { Flake } \\
\text { content } \\
(\%)\end{array}$ & $\begin{array}{c}\text { Dust } \\
\text { particles } \\
\text { content \% }\end{array}$ & $\begin{array}{c}\text { Weight } \\
\text { yield } \\
\%\end{array}$ & $\begin{array}{c}\text { Flake } \\
\text { content } \\
(\%)\end{array}$ & $\begin{array}{c}\text { Dust } \\
\text { particles } \\
\text { content \% }\end{array}$ & $\begin{array}{c}\text { Efficiency } \\
\mathrm{E}+ \\
(\%)\end{array}$ \\
\hline \multirow{2}{*}{ Steady grillage } & $0.2-0.7$ & 29,6 & 21 & 80,00 & 36,20 & 12 & 97,84 \\
\hline & $0.7-2.0$ & 26,0 & 4 & 72,00 & 33,50 & - & 92,80 \\
\hline \multirow{2}{*}{$\begin{array}{l}\text { Concentration sluice } \\
\text { with dry operation }\end{array}$} & $0.2-0.7$ & 29,6 & 21 & 78,10 & 36,50 & 10 & 96,30 \\
\hline & $0.7-2.0$ & 26,0 & 4 & 72,00 & 33,00 & - & 91,40 \\
\hline \multirow{2}{*}{$\begin{array}{l}\text { Electromagnetic } \\
\text { vibrational sieve }\end{array}$} & $0.2-0.7$ & 29,6 & 21 & 78,12 & 36,30 & 6,7 & 95,80 \\
\hline & $0.7-2.0$ & 26,0 & 4 & 71,85 & 32,40 & - & 89,50 \\
\hline \multirow{2}{*}{$\begin{array}{l}\text { Rebound drum } \\
\text { separator }\end{array}$} & $0.2-0.7$ & 29,6 & 21 & 70,65 & 35,50 & - & 84,70 \\
\hline & $0.7-2.0$ & 26,0 & 4 & 68,45 & 30,50 & - & 80,30 \\
\hline
\end{tabular}

$\mathrm{E}+=\beta . \gamma / \alpha$, where $\alpha$ - flake content of inputted raw material $(\%), \quad \beta$ - flake content of upgraded concentrate, $\gamma$ - weight yield of concentrate (\%); dust particles are below dimensions of $0.2 \mathrm{~mm}$ 
- drum-type rebound separator

From the reason of simplicity and plainness of equipment together with the necessary output, the most suitable results were obtained using the fixed slot grate or eventually its combination with the electromagnetic vibration screen. The results obtained are in Tab. 10. Accordingly, in the $0.2-0.7 \mathrm{~mm}$ size fraction, the amount of flakes increased from 29.6 to $36.2 \%$ at a $80 \%$ yeld and $97.8 \%$ efficiency. The amount of dust particles decreased from 21 to $12 \%$. The efficiency in the $0.5-2 \mathrm{~mm}$ size fraction is somewhat lower.

\section{Conclusion}

The talcose rocks of lower quality and rarely exploited rock varieties accompanying talc occurrences were tested for obtaining the high quality talc products.

The talc extraction and beneficiation from carbonatic talcose rocks was done using flotation method. The principal characteristicts of flotation and its products are tabled. The obtained products have practical use in pharmaceutical, electroceramic and rubbery industry.

The talc flakes, present in sericite-chlorite schistose rocks were tested for special use in paperboard for relatively untraditional roof's covering. According to the results indicated, the quality of rock suitable for flake production, applying grinding and sizing in dry state, was postulated. After the determination of the rock type, the relation between the sum of $\mathrm{Al}_{2} \mathrm{O}_{3}+\mathrm{MgO}+\mathrm{K}_{2} \mathrm{O}$ (in wt. \%) and the flake content is as follows (see table).

The magnesite concentrate, the by-product of talcose raw material dressing, was successfully used as an additive for wall-plaster substances. By this way all components of parent rock were used by human community and this approach fulfils the demand of environmentally friendly wasteless technology.

\begin{tabular}{|c|c|c|}
\hline $\mathrm{Al}_{2} \mathrm{O}_{3}+\mathrm{MgO}+\mathrm{K}_{2} \mathrm{O}$ (wt. \%) & \multicolumn{2}{|c|}{ flake content in } \\
\hline & $\begin{array}{c}\text { wype 1 - sericite-chlorite schist } \\
\text { (chlorite, sericite, resp. muscovite, } \\
\text { quartz) }\end{array}$ & $\begin{array}{c}\text { Type 2 - steatitized sericite-chlorite } \\
\text { schist (chlorite, sericite, resp. } \\
\text { muscovite, quartz, talc to 10 \%) }\end{array}$ \\
\hline under 15 & 0 & 0 \\
\hline $15-20$ & $0-13$ & $0-15$ \\
\hline $20-25$ & $13-28$ & $15-34$ \\
\hline $25-30$ & $28-45$ & $34-60$ \\
\hline $30-35$ & $45-65$ & $60-90$ \\
\hline
\end{tabular}

Note: The rock type 3 - strongly steatitized chlorite schist is for the flakes production unsuitable.

\section{References}

Bezák V. 1994. Suggestion of a new division of crystalline complexes of Western Carpathians based on reconstruction of Hercynian tectonic setting. Mineralia Slov., 26, 1-6. (In Slovak with English resume)

Bezák V. ed. 1999. Explanations to geological map of the Slovak ore-mountains - western part. Geological Survey of Slovak Republic. Dionýz Štúr publishers, Bratislava, 1-176. (In Slovak with English resume)

Derco J. 1986. Talc and talc-bearing rocks of the Sinec deposit (Central Slovakia) and possibilities of their use. Mineralia Slov., 18, 51-63. (In Slovak with English resume)
Derco J. \& Vlasák M. 1984. Flaky talc from Kokava nad Rimavicou (Central Slovakia) and methods of its preparation. Mineralia Slov., 16, 139-156. (In Slovak with English resume)

Grecula P., Radvanec, M. \& Németh Z. 2000. Magnesite and talc mineralization in Slovakia. Mineralia Slov., 32, 533-542.

Radvanec M. \& Prochaska W. 2001. Successive replacement of Upper Carboniferous calcite to dolomite and magnesite in Dúbrava magnesite deposit (Western Carpathians, Slovakia). Mineralia Slov., 33, 517-525. 